\title{
A relação entre demanda e desempenho dos magistrados: investigação de um modelo funcional em forma de U invertido
}

\author{
Renan Marçal Manzi ${ }^{1}$ \\ Marcos de Moraes Sousa 12 \\ 1 Universidade Federal de Goiás / Programa de Pós-Graduação em Administração, Goiânia / GO - Brasil \\ 2 Instituto Federal Goiano Campus Ceres / Programa de Pós-Graduação em Educação Profissional e Tecnológica ProfEPT, \\ Ceres / G0 - Brasil
}

\begin{abstract}
A hipótese da produtividade exógena aponta a relação entre quantidade de juízes e desempenho como linear, porém várias fragilidades vêm sendo apontadas, como o limite de produção dos juízes. Diante disso, o objetivo deste trabalho é testar se o desempenho dos juízes em relação à demanda tem um modelo funcional em forma de U invertido. Os dados usados na pesquisa compreendem os anos de 2009 a 2019. Os resultados foram estatisticamente significantes para todas as variáveis analisadas, tanto para a justiça estadual quanto para a trabalhista, confirmando a hipótese de que o desempenho e a demanda judicial podem responder a um modelo funcional em forma de U invertido. A confirmação da hipótese de pesquisa traz luz à discussão sobre como a pressão da demanda afeta o desempenho e como elas se relacionam. Este artigo discutiu e endossou as conclusões de outros autores sobre fragilidades e incongruências da hipótese da produtividade exógena para o Judiciário. Há evidências empíricas de que o aumento da demanda judicial pressiona o aumento de desempenho dos juízes, mas esse crescimento tem limites, e a partir de determinado ponto isso pode declinar e afetar negativamente o desempenho.

Palavras-chave: judiciário; desempenho; métodos quantitativos; carga de trabalho; administração pública.
\end{abstract}

\section{La relación entre la demanda y el desempeño de los magistrados: investigación de un modelo funcional en forma de $U$ invertida}

La hipótesis de la productividad exógena indica que la relación entre el número de jueces y el rendimiento es lineal, pero se han señalado varios puntos débiles, como el límite de producción de los jueces. En vista de ello, el objetivo de este trabajo fue comprobar para los tribunales estatales y laborales si la actuación del juez en relación con la demanda tiene un modelo funcional en forma de $U$ invertida. Los datos utilizados en la investigación abarcan los años 2009 a 2019. Los resultados fueron estadísticamente significativos para todas las variables analizadas tanto para los tribunales estaduales como para los laborales confirmando la hipótesis de que el rendimiento y la demanda judicial pueden responder a un modelo funcional en forma de $U$ invertida. La confirmación de la hipótesis de la investigación arroja luz sobre la discusión sobre cómo la presión de la demanda afecta al rendimiento y cómo se relacionan. La contribución de este trabajo fue discutir y refrendar las conclusiones de otros autores sobre las debilidades e incongruencias de la hipótesis de la productividad exógena del poder judicial. Hay pruebas empíricas de que el aumento de la demanda judicial ejerce presión sobre el aumento del rendimiento de los jueces, pero este crecimiento tiene límites y a partir de cierto punto puede disminuir y afectar negativamente al rendimiento. Palabras clave: poder judicial; desempeño; métodos cuantitativos; carga de trabajo; administración pública.

\section{The relationship between demand and performance of magistrates: investigation of a functional model in the form of an inverted $U$}

The hypothesis of exogenous productivity points to the relationship between the number of judges and performance as linear. However, several weaknesses have been pointed out, such as the production limit of the judges. Therefore, this work aims to test if the performance of judges related' to demand has an inverted U functional model. The data used in the research comprise the years from 2009 to 2019. The results were statistically significant for all variables analyzed for both state and labor courts, confirming the hypothesis that performance and judicial demand may respond to an inverted $U$ functional model. Confirming the research hypothesis sheds light on the discussion of how demand affects performance and how they relate. The contribution of this work lies in the discussion and endorsing the conclusions of other authors concerning weaknesses and incongruities of the hypothesis of exogenous productivity for the judiciary. There is empirical evidence that the increase in judicial demand pressures the increase in the performance of judges, but this increase has limits, and after a certain point, this can decline and negatively affect performance.

Keywords: judiciary; performance; quantitative methods; workload; public administration. 


\section{AGRADECIMENTOS}

Os autores agradecem ao Instituto Federal Goiano pelo apoio financeiro na tradução do artigo; à Fundação de Amparo à Pesquisa do Estado de Goiás (FAPEG) pela bolsa de formação a Renan Marçal Manzi e ao Conselho Nacional de Desenvolvimento Científico e Tecnológico no projeto CNPq (404709/2018-4).

\section{INTRODUÇÃO}

O Poder Judiciário é responsável por resolver os conflitos sociais por meio dos processos judiciais e vem sofrendo com a morosidade e o acúmulo de ações pendentes de julgamento, como evidenciado no relatório Justiça em Números, do Conselho Nacional de Justiça (CNJ, 2020). No fim de 2019, mais de 76 milhões de processos aguardavam alguma decisão judicial (CNJ, 2020). Esse número demonstra a dimensão do problema relacionado com a eficiência na prestação de serviços judiciais ao cidadão.

O baixo desempenho do Judiciário não é um problema apenas do Brasil; vários países ao redor do mundo passam por essa mesma dificuldade. Podem-se destacar os estudos que avaliaram o desempenho nos tribunais realizados em Portugal (Teixeira, Bigotte, Repolho \& Antunes, 2019), na Holanda (Blank \& Heezik, 2020), na Itália (Falavigna, Ippoliti \& Ramello, 2018), na Argentina (Ferro, Romero \& Romero-Gómez, 2018), na Bulgária (Dimitrova-Grajzl, Grajzl, Slavov \& Zajc, 2016), no Japão (Ramseyer, 2012), nos Estados Unidos (Christensen \& Szmer, 2012), na Espanha (RosalesLoópez, 2008), em Israel (Beenstock \& Haitovsky, 2004) e no Brasil (Gomes, Alves \& Silva, 2018; Gomes, Guimarães \& Akutsu, 2017; Procopiuck, 2018; Sousa \& Guimarães, 2018).

Essa quantidade de trabalhos, em diferentes países, ilustra que a preocupação com a eficiência e a morosidade do sistema de Justiça ao redor do mundo é recorrente e de grande importância social. No caso brasileiro, destaca-se ainda que a baixa eficiência desse sistema não é decorrente só do arcabouço jurídico; está ligada também a problemas de ordem administrativa, política e cultural (Ribeiro \& Rudiniki, 2016).

A alta demanda pela Justiça e as constantes pressões pela melhoria do desempenho do sistema judicial precisam ser mais bem compreendidas. Já se sabe que o aumento da demanda pelos tribunais produz um efeito positivo no desempenho deles (Beenstock \& Haitovsky, 2004; Rosales-Loópez, 2008), mas o que ainda não se conhece é se esse efeito é linear e produz efeitos constantes ou tem algum limite conhecido (Sousa \& Guimarães, 2018).

Apesar de ser contraintuitivo imaginar que aumentando a carga de trabalho também aumentará o desempenho do juiz, identificou-se que a produção e a eficiência deles têm caráter exógeno, ou seja, um aumento na carga de trabalho opera como estímulo exterior para que se produza mais e, dessa forma, se equilibre a relação entre entrada e saída dos processos. Esse fenômeno ficou conhecido como "hipótese da produtividade exógena" e se ancora em diversos estudos (Beenstock \& Haitovsky, 2004; Dimitrova-Grajzl et al., 2012, 2016; Rosales-López, 2008) que indicam que a relação entre demanda e desempenho do judiciário não tem limites e detém uma correlação linear.

Atualmente, discute-se se a demanda e o desempenho judicial estabelecem uma relação funcional linear, como propõe a hipótese da produtividade exógena (Beenstock \& Haitovsky, 2004), ou outro comportamento, por exemplo, que tenha relação com o crescimento do desempenho até algum ponto crítico e, a partir daí, com um declínio da produtividade do sistema de Justiça (Sousa \& Guimarães, 2018). 
A hipótese de produtividade exógena, aparentemente, tem falhas em suas premissas, como a de linearidade entre as variáveis "demanda" e "desempenho judicial" e a da perspectiva de aumento constante da produtividade dos juízes. É preciso lembrar que o crescimento no número de processos não tem limites conhecidos, pois depende do conjunto da sociedade. Por outro lado, a capacidade produtiva do juiz tem um limite natural, dados o cansaço físico e mental e a escassez de tempo (Gomes, 2018; Gomes et al., 2017; Jonski \& Mankowski, 2014).

Assim, há uma lacuna na literatura ao não dimensionar qual seria esse limite de desempenho diante do aumento da demanda. Sousa e Guimarães (2018) sugerem como lacunas a serem estudadas a análise da demanda do juiz, ponto que pode indicar uma inflexão ao aumento de desempenho dos magistrados, na tentativa de verificar a premissa de linearidade e o limite de produtividade.

O presente trabalho questiona as premissas da hipótese da produtividade exógena ao tentar verificar se a relação entre a demanda e o desempenho judicial responde a um modelo funcional quadrático. A demanda foi definida como variável independente, e o desempenho ao quadrado, como variável dependente. Tentou-se prever uma relação em forma de U invertido, em que o desempenho teria crescimento até determinado ponto crítico, a partir do qual haveria uma possível queda de produtividade. Dessa forma, podem-se verificar a linearidade e o crescimento da produtividade de modo constante.

Outro ponto importante a ser lembrado é que o tema "desempenho no Judiciário" começou a se desenvolver recentemente, pela corrente denominada law and economics. Apesar de um constante crescimento do interesse por essa área, ainda é reduzida a quantidade de pesquisadores brasileiros que se dedicam a esse campo de conhecimento, quando comparados com outras áreas que estudam desempenho no setor público (Gomes \& Guimarães, 2013; Louro, Santos \& Zanquetto, 2017; Sousa \& Guimarães, 2014)few theoretical and empirical studies are found in Brazilian Judiciary Courts' literature. In order to empirically identify which variables (IT investments, own or outsourced human capital.

Diante do quadro apresentado acima, percebeu-se a necessidade de estudar um modelo funcional que explique a relação entre demanda e desempenho aplicado ao judiciário e à realidade brasileira. Com base no problema identificado na literatura, o objetivo deste trabalho é testar se o desempenho do juiz em relação à demanda tem um modelo funcional em forma de U invertido.

\section{RELAÇÃO ENTRE DEMANDA E DESEMPENHO JUDICIAL}

Uma discussão presente nos estudos sobre desempenho no judiciário se refere a quais variáveis determinam os resultados de produtividade dos juízes e dos tribunais. Roussey e Soubeyran (2018) descrevem três conjuntos de índices de saídas de desempenho judicial: o primeiro, ligado ao tempo e ao número de processos baixados pelos juízes; o segundo, à qualidade das decisões; o terceiro, aos termos da legislação vigente.

Entre esses conjuntos de índices, o primeiro vem se destacando com um número crescente de estudos nos últimos anos, na tentativa de encontrar as relações entre as variáveis que interferem na produtividade dos tribunais, como os trabalhos de Bielen, Peeters, Marneffe e Vereeck (2018), Ferro et al. (2018), Gomes et al. (2018), Procopiuck (2018), e Sousa e Guimarães (2018). 
Uma relação em especial entre duas variáveis, porém, vem chamando a atenção e ganhando espaço nos estudos: o vínculo entre demanda judicial, caracterizada pela quantidade de processos que entram no sistema, e o número de pessoal, caracterizado pela quantidade de pessoas, sobretudo juízes, que atuam na resolução dos casos. Exemplos de estudos que verificaram a relação dessas variáveis na literatura são Beenstock e Haitovsky (2004), Dimitrova-Grajzl et al. (2012), Ferro et al. (2018), Gomes et al. (2017), Rosales-López (2008), e Sousa e Guimarães (2018).

Os resultados encontrados por essas análises indicam que há uma relação positiva entre o aumento da demanda e a produtividade dos juízes, ou seja, quando há um acréscimo na carga de trabalho dos magistrados, há variação positiva em seu desempenho. Beenstock e Haitovsky (2004) afirmam que isso acontece porque os juízes, quando pressionados pelo aumento da demanda, diminuem o tempo dedicado a cada caso, aumentando a produção e evitando, num primeiro momento, o aumento indiscriminado do estoque processual.

Beenstock e Haitovsky (2004) foram um dos primeiros autores a criar um modelo matemático para explicar a relação entre produtividade e carga de trabalho dos juízes, e os resultados encontrados ficaram conhecidos como "hipótese da produtividade exógena dos magistrados". Essa ideia defende que pressões externas que não estão sob o controle dos magistrados exercem fortes influências na produtividade deles. Essa pressão, segundo os autores, caracterizada pelo constante aumento da demanda por serviços judiciais e por uma rápida solução dos casos pelas partes envolvidas no litígio, influencia-os a aumentar a produção, a fim de que os estoques de processos não se elevem a níveis alarmantes. Essa noção foi confirmada por vários autores, como Dimitrova-Grajzl et al. (2012, 2016), Ferro et al. (2018), Gomes et al. (2017), Rosales-López (2008), Sousa e Guimarães (2018), como relatado acima.

Apesar de ser a vertente teórica dominante nos estudos quantitativos de desempenho dos tribunais, a hipótese de produtividade exógena dos juízes vem sendo criticada e contestada por alguns autores. Jonski e Mankowski (2014) questionaram e apontaram erro na modelagem elaborada por Beenstock e Haitovsky (2004) ao citarem que essa relação não é linear, motivo pelo qual há uma imprecisão metodológica na análise. Gomes (2018) aponta que não há linearidade na relação entre demanda e produtividade no Judiciário brasileiro, indicando, assim como Jonski e Mankowski (2014), que o desempenho dos juízes tem limites.

A demanda por serviços judiciais vem aumentando em todo o mundo (Bielen et al., 2018). Em alguns lugares, como no Brasil, o estoque de processos aguardando julgamentos chega à casa dos milhões, como constatado no relatório da Justiça em Números, do CNJ (2018). Isso evidencia que a carga de trabalho a que os juízes brasileiros são submetidos é muito alta e sem perspectiva de melhora. A cada ano, o estoque de processos pendentes de julgamento aumenta, como se pode verificar nos relatórios do CNJ de 2003 a 2018, e essa variável não tem nenhum limite conhecido até o momento. Por outro lado, a produtividade dos juízes tem limite, seja por fatores individuais, seja por limite de tempo.

Falavigna, Ippolite e Ramello (2018), Gomes (2018), Gomes et al. (2017), Jonski e Mankowski (2014) e Sousa e Guimarães (2018) lembram que há limite para o aumento da produtividade dos magistrados e que, a partir desse ponto, pode haver uma inflexão na produção. Gomes et al. (2017) apontam, com base nos dados do CNJ (2016), que a produtividade dos juízes começou a diminuir, evidenciando que o limite pode ter sido alcançado. 
Outro ponto a destacar é a tentativa, por parte dos governos, de aumentar o número de juízes efetivos para tentar diminuir o aumento dos estoques pendentes de julgamento. Autores como Beenstock e Haitovsky (2004), Dimitrova-Grajzl et al. (2012) e Gomes e Freitas (2017) evidenciaram que elevar o número de magistrados não aumenta a produtividade dos tribunais. A explicação para esse fenômeno é que os juízes sobrecarregados diminuem seu desempenho com a chegada dos novos magistrados, pois a carga de trabalho individual é virtualmente aliviada. Além de afetar negativamente a produtividade dos tribunais, o aumento do número de magistrados, se não acompanhado pelo crescimento do número de assistentes administrativos, poderia ter impacto negativo ainda maior, já que são esses assistentes que realizam a maior parte das atividades judiciais burocráticas (Gomes, 2018).

Muitos fatores influenciam o desempenho dos magistrados, como uso da tecnologia (Gomes et al., 2018), custos da Justiça (Blank \& Heezik, 2020), gestão administrativa (Roussey \& Soubeyran, 2018), experiência profissional dos juízes (Ferro et al., 2018) ou mesmo o tamanho da estrutura institucional (Peyrache \& Zago, 2016). Para algumas dessas variáveis, porém, ainda não há dados secundários estruturados ou estes não se moldaram ao escopo deste estudo, que focou no desempenho pela influência da demanda judicial já consolidada na literatura da área.

\subsection{Hipótese de Pesquisa}

A hipótese desta pesquisa foi elaborada com base em dois pressupostos retirados da literatura da área de desempenho no judiciário e apresentados a seguir. O primeiro tem como referência Dimitrova-Grajzl et al. (2012, 2016), Ferro et al. (2018), Gomes et al. (2017); Rosales-López (2008), e Sousa e Guimarães (2018), que afirmam haver uma relação positiva entre carga de trabalho e produtividade dos juízes. Com isso, formulou-se a primeira hipótese: "A produção dos juízes aumenta com a demanda judicial".

Apesar de este trabalho assumir que a produtividade aumenta com a demanda, Gomes (2018), Gomes et al. (2017), Jonski e Mankowski (2014), e Sousa e Guimarães (2018) lembram que esse aumento tem um limite, que essa relação encontrada nos estudos anteriores não é infinita e que deve haver um ponto de inflexão do nível de desempenho. Assim, formulou-se a segunda hipótese: "A produção dos juízes tem um limite superior de desempenho".

Baseado nas hipóteses preliminares formuladas acima, pretendeu-se investigar e testar a seguinte hipótese de pesquisa: "A produtividade dos juízes segue uma função em forma de U invertido (função quadrática) quando submetidos ao aumento da carga de trabalho".

\section{METODOLOGIA}

Não foi encontrado na revisão da literatura nenhum outro estudo com algum modelo empírico teórico que tivesse o mesmo objetivo ou usado a metodologia aqui escolhida. O teste de um modelo quadrático para verificar a correlação entre desempenho e carga de trabalho judiciária decorre de lacunas de pesquisas sugeridas por autores como Sousa e Guimarães (2018), ao indicarem ser preciso encontrar, caso exista, um ponto de otimização dessa relação. O modelo quadrático poderia, em tese, ajudar nessa busca. O que há de mais próximo são os estudos de Peyrache e Zago (2016), além de Santos e Amado (2014), que tentaram encontrar um valor ideal para tamanho e quantidade de tribunais (Voigt, 2012), ou para ambos (Teixeira et al., 2019), e não para a relação entre carga de trabalho e produtividade.Diante do que foi discutido na elaboração das hipóteses, o modelo que 
melhor atende aos pressupostos para atingir o objetivo da pesquisa se daria por regressão quadrática. Segundo Agresti e Finlay (2012), os modelos de regressão ordinários assumem que a relação entre as variáveis estudadas é linear, embora nas ciências sociais essas relações não sejam lineares. No entanto, graças ao baixo grau de não linearidade, elas podem ser aproximadas e estudadas como lineares. Ainda de acordo com os autores, esse modelo pode ser inadequado para modelar variáveis em que há relação de não linearidade.

Agresti e Finlay (2012) afirmam que consequências indesejáveis podem aparecer ao estimar um modelo linear de modo a explicar um fenômeno curvilíneo. Estimativas das médias das variáveis, na regressão linear, podem ser tendenciosas e levar a um distanciamento da linha que melhor representaria a relação estudada. Dessa forma, modelos de correlação podem não ser estimados da melhor maneira possível.

Wooldridge (2010) esclarece que adicionar termos quadráticos em variáveis significantes pode ser uma forma de verificar e encontrar o melhor modelo funcional de uma relação entre variáveis. O autor afirma que, apesar de complicar o entendimento do modelo funcional, termos quadráticos podem representar outros problemas funcionais ainda não conhecidos.

Werkema (1996) demonstra que uma função de regressão polinomial para uma variável resposta (y) e uma variável explicativa única $(\mathrm{x})$ tem a seguinte forma geral:

$$
\gamma=\beta_{0}+\beta_{1} \mathrm{X}+\beta_{2} \mathrm{X}^{2}+\cdots+\beta_{k} \mathrm{X}^{k} \varepsilon
$$

O valor do $k$ corresponde ao grau da equação. Se $(k=1)$, tem-se uma regressão linear. Para $(k=2)$, tem-se uma função de segundo grau, mais conhecida como regressão quadrática. De acordo com o autor, a função polinomial de segundo grau tem a seguinte função:

$$
\gamma=\beta_{0}+\beta_{1} \mathrm{X}+\beta_{2} \mathrm{X}^{2}+\varepsilon
$$

Na fórmula acima, $\beta_{0}$ é a média de y quando $\mathrm{x}=0$, $\beta_{1}$ é o parâmetro associado ao efeito linear de $\mathrm{x}, \beta_{2}$ é o parâmetro associado ao efeito quadrático e é o termo de erro associado às falhas do modelo em relacionar perfeitamente os dados. Com esse modelo estatístico, propõe-se o teste de regressão entre a produtividade e a carga de trabalho dos juízes estaduais e trabalhistas do Brasil, de modo a verificar sua aderência aos dados disponibilizados pelo CNJ (2018). O modelo funcional testado é indicado a seguir:

$$
\begin{aligned}
\text { Produção }= & \text { Carga de trabalho }+ \text { Carga de trabalho }{ }^{2} \\
& + \text { Assistentes administrativos }+ \text { Termo de erro } \\
\text { Prod }= & \beta_{0}+\beta_{1} \text { Carg }+\beta_{2} \text { Carg }^{2}+\beta_{3} \text { Admin }+\varepsilon
\end{aligned}
$$

As principais variáveis usadas nos mais recentes estudos de desempenho no judiciário são: casos novos, casos pendentes, casos resolvidos, número de juízes, número de audiência, investimento em tecnologia da comunicação e da informação, número de assistentes e equipes de apoio, custo total. 
Devido à dificuldade de acesso ou à não existência dos dados referentes a todas essas variáveis em tribunais estaduais e trabalhistas, este trabalho utilizou as variáveis "carga de trabalho", "assistentes administrativos" e "produção do juiz". No Quadro 1, são exibidas as variáveis e as referências que embasam essa escolha.

\section{QUADRO 1 VARIÁVEIS DA PESQUISA}

\begin{tabular}{lcc} 
Indicador & Variável & Referência bibliográfica \\
\hline Variável independente & Carga de trabalho (carg.) & Ferro et al. (2018), Gomes et al. (2017), \\
Variável independente & Assistentes administrativos (admin.) & Gomes et al. (2018), Rosales-López (2008). \\
Variável dependente & Produção do juiz (prod.) &
\end{tabular}

Fonte: Elaborado pelos autores.

A variável "carga de trabalho" representa o número total de processos judiciais submetidos aos tribunais dividido pelo número de juízes que cada um tem, podendo ser analisada o estoque de processo total ou em faixas temporais. É composta por subvariáveis “novos processos", que são inseridos no início de determinado período de análise, e "processos pendentes", que já foram inseridos num momento anterior à análise e que aguardam decisão do juiz.

Como apontado por Yeung e Azevedo (2011), a ponderação da carga de trabalho pelo número de juízes é indicada para controlar as variações do tamanho entre os tribunais, uma vez que a carga de trabalho oscila livremente, enquanto o número de juízes é fixado em lei e permanece relativamente constante ao longo do tempo. Portanto, adotar a média a que cada juiz de determinado tribunal é submetido minimiza os problemas relacionados com as diferenças de tamanho e estrutura entre os tribunais no país. Essa mesma estratégia também foi adotada por Gomes et al. (2018) para relativizar e minimizar as diferenças entre os tribunais que apresentavam discrepâncias na demanda por serviços judiciais e nos recursos disponíveis (físicos, financeiros e pessoais) para execução de suas atividades.

A variável "assistente administrativo" representa o número de pessoas disponíveis para as atividades administrativas que auxiliam os tribunais dividido pelo número de juízes de cada tribunal. Aqui, não há diferenciação do tipo e do grau de dificuldade das atividades, sendo contabilizada exclusivamente a quantidade de pessoas que cada juiz tem à disposição.

A variável "produção do juiz" representa o número de processos judiciais analisados e julgados pelos tribunais dividido pelo número de juízes de cada um e que foram baixados em determinado período temporal, ou seja, que esgotaram seu trâmite nas competências de cada magistrado ou tribunal.

Os dados usados na pesquisa são de origem secundária, coletados na base Justiça em Números, pelo CNJ (2020), e disponibilizados para utilização e download no sítio eletrônico da instituição. Eles foram colhidos desde 2005, porém a partir de 2009 foi estabelecida uma nova metodologia para a coleta. Tem-se hoje um banco de dados em painel com informações empilhadas separadas por tribunal e por ano, com variáveis numéricas (contínuas e discretas) e categóricas. 
O presente estudo tem duas variáveis, que, no banco de dados do CNJ, correspondem a três subvariáveis. A variável "carga de trabalho" corresponde à soma das subvariáveis "cn1", que significam casos novos para o primeiro grau de jurisdição, e "cp1", casos pendentes para o primeiro grau de jurisdição. A variável "produção do juiz" corresponde a subvariável "tbaixl”, que é o total de processos baixados para o primeiro grau de jurisdição. A variável "assistente administrativo" corresponde a subvariável "sajud1", também relativa ao primeiro grau de jurisdição. As variáveis deste estudo foram ponderadas pelo número de juízes providos (subvariável "magp 1") em cada tribunal, com o objetivo de diminuir as variações em decorrência das diferenças de estrutura e tamanho do judiciário de cada região e estado da federação.

Todas as variáveis são numéricas, discretas, e não há dados faltantes para elas, deixando o painel balanceado. Os dados correspondem aos anos de 2009 a 2019, pois nos anteriores a metodologia e as variáveis colhidas pelo estudo eram diferentes.

Para a análise de dados, usou-se o modelo funcional proposto neste trabalho. Como constatado em Hill, Griffiths e Judge (2006), não existem regras claras que ajudem a escolher qual modelo funcional entre variáveis é o que melhor representa a relação estudada. Isso depende dos problemas teóricos inseridos na discussão da problemática ou da pergunta de pesquisa, dos dados disponíveis para análise ou da capacidade do pesquisador em compreender qual interação responderia aos objetivos do tema testado.

Relações lineares, contudo, já foram encontradas na literatura, como discutido na parte teórica, e há indícios de uma possível relação quadrática dessas variáveis que, potencialmente, poderia melhor representar essa correlação.

Em estudos de análise de regressão, um dos objetivos para a elaboração funcional da relação é obter estimativas estatisticamente significantes de valores e sinais dos regressores do modelo que permitam fazer inferências estatísticas e atendam à base teórica do fenômeno estudado (Gujarati \& Porter, 2011).

A segunda etapa foi analisar de que forma a regressão com dados em painel seria realizada. Há três técnicas mais comuns para a realização de regressão com dados em painel: pooled ordinary least squares (Pols), modelo de efeitos fixos (MEF) e modelo de efeitos aleatórios (MEA). Realizou-se o teste Hausman para decidir entre as técnicas MEA e MEF, o teste F restrito para decidir entre Pols e MEF e o teste lagrange multiplier de Breusch-Pagan para decidir entre Pols e MEA. Esses testes pretendem verificar se os estimadores dos dois modelos em comparação diferem ou não substancialmente um do outro e indicar o mais adequado (Fávero, Belfiore, Silva \& Chan, 2009). As hipóteses e os valores dos resultados dos testes para a escolha do modelo de regressão serão apresentados na seção de resultados.

O método de cálculo dos estimadores depende do modelo de regressão selecionado. Para o modelo de efeito fixo e pooled ordinary least squares, foi utilizado o método de mínimos quadrados ordinários (MQO), enquanto para o modelo de efeito aleatório foi utilizado o método de mínimos quadrados generalizados (MQG), já que para esse modelo o MQO produz estimadores ineficientes (Gujarati \& Porter, 2011; Maddala, 2003).

Usou-se o software "RStudio", versão 1.2.5001, para os cálculos estatísticos. A opção por esse software se deu por sua gratuidade e pelas opções de criar e executar diferentes testes e modelos que poderiam ser utilizados neste estudo. 


\section{RESULTADOS}

Inicialmente, foram realizados testes para avaliar os pressupostos básicos do modelo clássico de regressão. O Quadro 2 sistematiza os testes para avaliar se há problemas de heterocedasticidade, autocorrelação, multicolinearidade e normalidade dos resíduos para os dados da justiça estadual e trabalhista.

\section{QUADRO 2 RESULTADOS DAS PREMISSAS PARA A JUSTIÇA ESTADUAL E TRABALHISTA}

\begin{tabular}{|c|c|c|c|}
\hline \multicolumn{4}{|c|}{ Justiça estadual } \\
\hline Premissa & Teste & Resultado & Diagnóstico \\
\hline Heterocedasticidade & Breusch-Pagan & $\begin{array}{c}\mathrm{BP}=12,89 ; \\
\mathrm{p} \text {-valor=0,004 }\end{array}$ & Não atende à premissa \\
\hline Autocorrelação & Durbin-Watson & $\begin{array}{c}D W=2,09 ; \\
p-\text { valor=0,801 }\end{array}$ & Atende à premissa \\
\hline Multicolinearidade & VIF & De 1,20 a 11,34 & Não atende à premissa \\
\hline Normalidade & Shapiro-Wilk & $\begin{array}{c}W=0,96 ; \\
p-\text { valor }=0,001\end{array}$ & Não atende à premissa \\
\hline \multicolumn{4}{|c|}{ Justiça trabalhista } \\
\hline Premissa & Teste & Resultado & Diagnóstico \\
\hline Heterocedasticidade & Breusch-Pagan & $\begin{array}{c}\mathrm{BP}=13,48 ; \\
\text { p-valor=0,003 }\end{array}$ & Não atende à premissa \\
\hline Autocorrelação & Durbin-Watson & $\begin{array}{c}D W=1,97 ; \\
\text { p-valor }=0,409\end{array}$ & Atende à premissa \\
\hline Multicolinearidade & VIF & De 1,04 a 32,60 & Não atende à premissa \\
\hline Normalidade & Shapiro-Wilk & $\begin{array}{c}W=0,98 \\
p-v a l o r=0,002\end{array}$ & Não atende à premissa \\
\hline
\end{tabular}

Fonte: Elaborado pelos autores.

Apesar de os modelos não atenderem ao pressuposto de heterocedasticidade, é possível corrigir esse problema modificando o método de estimação dos parâmetros. Quando há heterocedasticidade, os estimadores MQO deixam de ser eficientes, o que pode ser corrigido adotando os estimadores MQG, que, nessa situação, produzem melhores estimadores lineares não tendenciosos (Gujarati \& Porter, 2011).

Os dados indicam a não existência de autocorrelação dos termos de erro nos dados e no modelo proposto neste trabalho, atendendo à premissa do modelo clássico de regressão. 
A multicolinearidade, nesse caso, foi provocada pela adição da variável "carga de trabalho ao quadrado" ao modelo funcional, provocando uma correlação perfeita entre as duas variáveis. A adição de termos polinomiais a um modelo de regressão pode provocar o aparecimento da multicolinearidade, e uma das formas de lidar com esse problema é não fazer nada, pois mesmo assim é possível fazer estimações com relativa eficiência (Gujarati \& Porter, 2011).

É preciso ressaltar que a não normalidade dos resíduos se deve à própria estrutura de dados. Para resolver esse problema, recorreu-se à lei dos grandes números, por meio de teorema da teoria da probabilidade, ou seja, à medida que o tamanho da amostra cresce, a distribuição dos erros dos resíduos se aproxima da distribuição normal, produzindo estimadores válidos (Gujarati \& Porter, 2011). Para cada variável do estudo, foram usadas 297 observações para a justiça estadual e 264 para a trabalhista.

Após os testes dos pressupostos, iniciaram-se os testes para a escolha dos modelos de estimação. O Quadro 3 sistematiza os testes e a indicação de qual modelo seria mais apropriado para a base de dados utilizadas no presente estudo.

\section{QUADRO 3 RESULTADOS DA ESCOLHA DO MODELO DE ESTIMAÇÃO}

\begin{tabular}{|c|c|c|c|c|}
\hline \multicolumn{5}{|c|}{ Justiça estadual } \\
\hline Teste & Resultado & P-valor & Hipóteses & Diagnóstico \\
\hline Teste F & 1,3294 & 0,1366 & $\begin{array}{l}\mathrm{HO}=\text { Pols } \\
\mathrm{H} 1=\mathrm{MEF}\end{array}$ & Pols \\
\hline $\begin{array}{l}\text { Teste lagrange multiplier de Breusch- } \\
\text { Pagan }\end{array}$ & 0,9653 & 0,3258 & $\begin{array}{l}\mathrm{H} 0=\text { Pols } \\
\mathrm{H} 1=\mathrm{MEA}\end{array}$ & Pols \\
\hline Teste de Hausman & 2,6167 & 0,4546 & $\begin{array}{l}\mathrm{H} 0=\mathrm{MEA} \\
\mathrm{H} 1=\mathrm{MEF}\end{array}$ & MEA \\
\hline \multicolumn{5}{|c|}{ Justiça trabalhista } \\
\hline Teste & Resultado & P-valor & Hipóteses & Diagnóstico \\
\hline Teste F & 0,7845 & 0,7493 & $\begin{array}{l}\mathrm{HO}=\text { Pols } \\
\mathrm{H} 1=\mathrm{MEF}\end{array}$ & Pols \\
\hline $\begin{array}{l}\text { Teste lagrange multiplier de Breusch- } \\
\text { Pagan }\end{array}$ & 0,7950 & 0,3726 & $\begin{array}{l}\mathrm{H} 0=\text { Pols } \\
\mathrm{H} 1=\mathrm{MEA}\end{array}$ & Pols \\
\hline Teste de Hausman & 4,8014 & 0,1869 & $\begin{array}{l}\mathrm{H} 0=\mathrm{MEA} \\
\mathrm{H} 1=\mathrm{MEF}\end{array}$ & MEA \\
\hline
\end{tabular}

Fonte: Elaborado pelos autores. 
Após essa série de teste para a escolha do modelo de regressão, o modelo pooled ordinary least squares foi o mais indicado, sendo, portanto, usado nas regressões para calcular os melhores estimadores para o modelo funcional testado neste trabalho, tanto para a justiça estadual quanto para a trabalhista. Os dados de ambas as justiças apontaram heterocedasticidade, e uma das formas de corrigir esse problema é usando estimadores com a técnica dos mínimos quadrados generalizados (MQG), usado apenas no modelo de efeitos aleatórios (MEA). Por essa limitação, foi selecionado o modelo de efeitos aleatórios (MEA) para a análise dos dados neste trabalho.

A seguir, a Tabela 1 apresenta os resultados da regressão do modelo funcional proposto neste estudo em relação à justiça estadual. Foi utilizado o modelo de efeitos aleatórios (MEA), e os estimadores foram calculados pelo método dos mínimos quadrados generalizados. Como já citado, os dados em painel estavam balanceados, totalizando 297 informações distintas para cada variável.

TABELA 1 RESULTADOS DE REGRESSÃO MÚLTIPLA DA JUSTIÇA ESTADUAL

\begin{tabular}{|c|c|c|c|c|c|}
\hline \multicolumn{6}{|c|}{ Prod $=\beta_{0}+\beta_{1}$ Carg $+\beta_{2}$ Carg $^{2}+\beta_{3}$ Adimin $+\varepsilon$} \\
\hline & Coeficiente & Erro padrão & Razão-t & P-valor & Sig. \\
\hline Constante & 169,84 & 92,83 & 1,8295 & 0,0673 & \\
\hline Carga & 0,1531 & 0,0182 & 8,3889 & $<0,0001$ & *** \\
\hline Carga $^{2}$ & $-0,0000029$ & 0,0000009 & $-2,9195$ & 0,0035 & ** \\
\hline Assistente & 26,694 & 6,266 & 4,2600 & $<0,0001$ & $\star \star \star ~$ \\
\hline$R^{2}: 0,5928$ & & \multicolumn{4}{|c|}{ Estatística F (3,293): 142,605 } \\
\hline$R^{2}$ ajustado: 0,5886 & & & \multicolumn{3}{|c|}{ P-valor: $<0,0001$} \\
\hline
\end{tabular}

Nota: Sig. = Nível de significância; ${ }^{\star} \mathrm{p}$-valor $<0,05$; ${ }^{* *} \mathrm{p}$-valor $<0,01$; ${ }^{* *} \mathrm{p}$-valor $<0,001 . \mathrm{R}^{2}=$ Coeficiente de determinação. gl $=$ grau de liberdade.

Intervalo de Confiança do coeficiente (99\%): -0,0000055 > > -0,00000041.

Fonte: Elaborado pelos autores.

Os resultados foram estatisticamente significantes para todas as variáveis analisadas. A variável "carga" teve como estimativa o valor de $\beta_{1}=0,1531 \mathrm{com}$ p-valor $<0,0001$, indicando significância para o modelo analisado. A variável "carga" teve como estimativa o valor de $\beta_{2}=-0,0000029$ com p-valor $=0,0035$, indicando significância para o modelo analisado. A variável "assistente" teve como estimativa o valor de $\beta_{3}=26,694$ com p-valor $<0,0001$, também indicando significância estatística para o modelo analisado. $\mathrm{O}$ teste $\mathrm{F}$ foi significativo para a aderência do modelo com estimativa $\mathrm{F}=142,60$ e p-valor $<0,0001$. A medida que indica o ajustamento generalizado do modelo conhecido como $\mathrm{R}^{2}$ ficou em 0,5928 , com $\mathrm{R}^{2}$ ajustado em 0,5886 . As estimativas encontradas podem indicar a possibilidade de o modelo teórico proposto neste trabalho ter sustentação nos dados da justiça estadual de primeiro grau brasileira. O estimador $\beta_{2}$ é significante e negativo, indicando uma curva funcional com concavidade para baixo, de acordo com o modelo testado.

Com sustentação nos dados do CNJ (2020) e no modelo proposto, infere-se que a hipótese de produtividade dos juízes pode ter uma função em forma de U invertido (função quadrática) quando submetida ao aumento da carga de trabalho. Ela, portanto, não pode ser rejeitada quando analisados os dados da justiça estadual. 
A Tabela 2 apresenta os resultados da estimação para a justiça trabalhista. Foi utilizado o modelo de efeitos aleatórios (MEA), e os estimadores foram calculados pelo método dos mínimos quadrados generalizados. Como já citado, os dados em painel estavam balanceados, totalizando 264 informações distintas para cada variável analisada.

TABELA 2 RESULTADOS REGRESSÃO MÚLTIPLA DA JUSTIÇA TRABALHISTA

\begin{tabular}{|c|c|c|c|c|c|}
\hline \multicolumn{6}{|c|}{ Prod $=\beta_{0}+\beta_{1}$ Carg $+\beta_{2}$ Carg $^{2}+\beta_{3}$ Adimin $+\varepsilon$} \\
\hline & Coeficiente & Erro padrão & Razão-t & P-valor & Sig. \\
\hline Constante & $-342,27$ & 136,52 & $-2,5291$ & 0,0114 & * \\
\hline Carga & 0,8004 & 0,1063 & 7,5244 & $<0,0001$ & 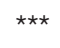 \\
\hline Carga $^{2}$ & $-0,0001165$ & 0,0000227 & $-5,1248$ & $<0,0001$ & $\star \star \star \star ~$ \\
\hline Assistente & 31,281 & 11,180 & 2,7979 & 0,0051 & $\star \star$ \\
\hline$R^{2}: 0,4936$ & & \multicolumn{4}{|c|}{ Estatística F $(3,260): 84,495$} \\
\hline $\mathrm{R}^{2}$ ajustado: 0,4878 & & & \multicolumn{3}{|c|}{ P-valor: $<0,0001$} \\
\hline
\end{tabular}

Nota: Sig. = Nível de significância; ${ }^{\star}$ p-valor $<0,05 ;{ }^{* *}$-valor $<0,01$; ${ }^{* * *}$-valor $<0,001 . \mathrm{R}^{2}=$ Coeficiente de determinação. gl $=$ grau de liberdade.

Intervalo de Confiança do coeficiente (99\%): -0,000175 > > -0,000057.

Fonte: Elaborado pelos autores.

Os resultados foram estatisticamente significantes para todas as variáveis analisadas. A variável "carga" teve como estimativa o valor de $\beta_{1}=0,8004$ com p-valor $<0,0001$, indicando significância estatística para o modelo analisado. A variável "carga" teve como estimativa o valor de $\beta_{2}=-$ 0,0001165 com p-valor $<0,0001$, indicando significância para o modelo analisado. A variável "assistente" teve como estimativa o valor de $\beta_{3}=31,281 \mathrm{com}$-valor $=0,0051$, também indicando significância estatística para o modelo analisado. $\mathrm{O}$ teste $\mathrm{F}$ foi significativo para a aderência do modelo com estimativa $\mathrm{F}=84,49 \mathrm{e}$-valor $<0,0001$. A medida que indica $\mathrm{o}$ ajustamento generalizado do modelo conhecido como $\mathrm{R}^{2}$ ficou em 0,4936 , e o $\mathrm{R}^{2}$ ajustado ficou em 0,4878 . As estimativas encontradas podem indicar a possibilidade de o modelo teórico proposto neste trabalho também ter sustentação nos dados da justiça trabalhista de primeiro grau brasileira. O estimador $\beta_{2}$ é significante e negativo, indicando uma curva funcional com concavidade para baixo, de acordo com o modelo testado.

Com sustentação nos dados do CNJ (2020) e no modelo proposto, infere-se que a hipótese de produtividade dos juízes pode ter uma função em forma de U invertido (função quadrática) quando estes são submetidos ao aumento da carga de trabalho. Dessa forma, a hipótese proposta não pode ser rejeitada quando analisados os dados da justiça trabalhista. 


\subsection{Discussão dos resultados}

Os resultados que emergem do presente trabalho confirmam a hipótese de que o desempenho e a demanda judicial podem responder a um modelo funcional em forma de U invertido, tanto para a justiça trabalhista quanto para a estadual brasileira. A não rejeição da hipótese de pesquisa proposta lança luz na discussão sobre como a pressão da demanda afeta o desempenho dos tribunais e como elas se relacionam. A discussão sobre a linearidade entre essas variáveis ganha novos contornos com o indicativo de que pode haver um limite de produtividade dos magistrados quando estes são submetidos às pressões advindas do aumento da carga de trabalho.

Outra constatação que emerge desses resultados diz respeito à discussão sobre até que ponto se pode aumentar a produtividade dos juízes, se há ou não um limite para esse aumento. Gomes (2018) iniciou a discussão no Brasil sobre se os magistrados já estão ou não nesse limite de desempenho. Ao analisar os dados descritivos desta pesquisa, parece haver uma convergência para concluir que os juízes da justiça estadual e trabalhista podem estar no limite, ou muito próximo do limite, de sua produtividade, por isso a pressão da demanda pode ter pouca influência no desempenho dos magistrados.

A variável "assistente administrativo" demonstrou ser uma muito importante na composição do desempenho dos juízes, como já previam Ferro et al. (2018), Gomes et al. (2017), Gomes et al. (2018), Rosales-López (2008), e Santos e Amado (2014), demonstrando talvez ser até mais significante do que a carga de trabalho na resposta da produtividade dos magistrados. Assim, deve-se pensar na mudança do nível de relevância da variável nas modelagens funcionais. A importância dessa variável na produtividade dos juízes vai ao encontro do que foi apontado por Gomes et al. (2017), que concluíram que a contratação de auxiliares para os magistrados é uma alternativa mais vantajosa para aumentar o desempenho do que contratações de juízes. Contudo, um número excessivo de assistentes não promove variação positiva no desempenho, e talvez haja algum ponto de equilíbrio entre desempenho e demanda dessa variável que ainda precise ser mais bem estudado.

Os resultados indicam que os dois principais ramos da justiça brasileira respondem a uma função quadrática entre produtividade e carga de trabalho. As estimativas do termo angular $\left(\beta_{1}\right)$ foram estatisticamente significantes, e o sinal negativo indica uma função com concavidade para baixo, evidenciando que pode haver ponto máximo da função ou ponto crítico. Esse possível ponto crítico, que seria o ponto ótimo do desempenho, demonstra a possibilidade teórica de um ponto real de inflexão da produtividade (variável de resposta) a partir do qual poderia até diminuir o desempenho, como supõe Sousa e Guimarães (2018).

Apesar de estatisticamente significante, o valor de tem baixa magnitude, talvez indicando que, a partir de determinado ponto, a variação do desempenho passe a variar de forma marginal, e não como uma relação direta. As críticas ao modelo linear de produtividade reforçam que a linearidade não é a relação mais adequada, sendo o modelo aqui exposto um primeiro passo para encontrar aquele que melhor se adéque aos dados empíricos.

Os resultados sugerem que há limites na produtividade dos juízes trabalhistas e estaduais. Mesmo que a demanda judicial aumente e pressione positivamente o desempenho, esse aumento não será linear. Nesse caso, será quadrático, indo de encontro com o que prevê a hipótese da produtividade exógena. Gomes (2018) já indicava que a relação entre desempenho e demanda na justiça brasileira não poderia seguir uma correlação linear, dada a diferença de características de cada variável. 
A suposição de linearidade da relação das variáveis estudadas também é colocada em dúvida neste estudo, pois supor que a demanda influenciará positivamente o desempenho e não prever algum limite é como supor que os magistrados poderão aumentar sua produtividade ad aeternum. Autores como Falavigna et al. (2018), Gomes (2018), Gomes et al. (2017), Jonski e Mankowski (2014), e Sousa e Guimarães (2018), já indicaram essa impossibilidade.

Há razões suficientes para questionar as premissas da hipótese da produtividade exógena. Jonski e Mankowski (2014), um dos primeiros autores a questionar a validade metodológica das premissas dessa hipótese, vêm ganhando companhia nos últimos anos. Falavigna et al. (2018), Gomes (2018), Gomes et al. (2017), e Sousa e Guimarães (2018) também questionam esses pressupostos e indicam que a produtividade de juízes pode apresentar limites humanos de desempenho, na mesma linha do que foi encontrado neste estudo.

Podem-se, com base nessas descobertas sobre a possível relação quadrática entre as variáveis "desempenho" e "demanda", encontrar mecanismos que permitam modelar um ponto de otimização do desempenho baseado demanda. Dessa forma, seria possível definir políticas de contratação de juízes e auxiliares segundo critérios mais objetivos, já que o recrutamento e a seleção de magistrados com o intuito de aumentar o desempenho e, assim, diminuir o número de processos que aguardam julgamento não surtirão efeito na produtividade dos tribunais, como apontado por Beenstock e Haitovsky (2004), Dimitrova-Grajzl et al. (2012), e Gomes e Freitas (2017). Os juízes sobrecarregados diminuem a produtividade com a chegada de novos magistrados, já que a carga de trabalho individual é virtualmente aliviada.

Os resultados gerais desta pesquisa sugerem que a hipótese da produtividade exógena precisa ser revista e atualizada, pois uma série de estudos vem apontando falhas metodológicas e teóricas em seus pressupostos. Já há indícios suficientes para apontar o limite de produção dos juízes e que o aumento indefinidamente da demanda a partir de determinado ponto tem pouco ou nenhum efeito prático no aumento do desempenho dos magistrados. A metodologia escolhida neste trabalho foi conduzida no sentido de tentar estimar um modelo funcional que suponha empiricamente um ponto de limite da produtividade dos juízes por meio de um ponto crítico de uma função quadrática.

Por fim, propõe-se que a relação entre desempenho e demanda dos tribunais possa ser analisadas sob uma perspectiva de não linearidade das variáveis e de um limite humano de desempenho dos magistrados. Este trabalho propõe que essa relação seja considerada um modelo funcional em forma de U invertido.

\section{CONCLUSÃO, LIMITAÇÕES E AGENDA DE PESQUISA}

Os estudos sobre desempenho no Judiciário começam a ganhar força, e um novo campo de estudo se estrutura no Brasil, no intuito de desenvolver respostas para os vários problemas de acesso e solução de conflitos por meio do Judiciário. Na balança entre oferta e demanda da justiça, há, de um lado, a sociedade, que demanda a solução de seus problemas, e, de outro, a oferta, a cargo do Estado, por meio de um sistema de mediação de conflitos que se concentra na figura do juiz.

Há uma relação direta entre demanda e oferta da justiça, e entender como essa correlação se comporta foi o problema investigado neste trabalho. O objetivo foi testar se a correlação entre desempenho e demanda judicial responde a um modelo funcional em forma de U invertido. Os resultados encontrados apontam para uma possível modelagem funcional que possa contemplar 
variáveis quadráticas. Como resultado, tem-se que um modelo funcional em forma de U invertido parece adequado para explicar a correlação entre demanda e produtividade judicial, pois os estimadores foram estatisticamente significantes para a justiça estadual e trabalhista.

A contribuição deste trabalho foi discutir e endossar as conclusões de outros autores, como Falavigna et al. (2018), Gomes (2018), Gomes et al. (2017), Jonski e Mankowski (2014), e Sousa e Guimarães (2018), sobre falhas e incongruências da hipótese da produtividade exógena para o Judiciário. É verdadeiro que o aumento da demanda judicial pressiona o aumento de desempenho dos juízes, mas esse acréscimo tem limites, e a partir de determinado ponto isso pode até prejudicar e afetar negativamente a produtividade. Há indicativos suficientes dos limites na produção dos magistrados, e os resultados aqui reportados corroboram essa afirmação. A hipótese de produtividade exógena precisa ser revista para prever a existência desse limite humano, independentemente de outras tecnologias que, porventura, possam ser adicionadas a esta equação entre demanda e desempenho.

Como limitações do estudo, pode-se indicar a utilização reduzida de variáveis no modelo funcional, pois a ausência de outras variáveis com influência no desempenho dos juízes pode levar a inconsistências na estimação dos parâmetros. Essa ausência de variáveis se deve a outra limitação encontrada: a dificuldade de acessar dados para além das variáveis levantadas pelo CNJ. Variáveis como "experiência do juiz", "número de comarcas de trabalho" (Gomes et al., 2017), "tempo alocado pelo magistrado em atividades administrativas" (Rosales-López, 2018; Roussey \& Soubeyran, 2018), entre outras que, reconhecidamente, influenciam o desempenho do juiz, ainda não têm dados estruturados disponíveis no Brasil, de modo que sua aquisição muitas vezes esbarra na burocracia dos tribunais, às vezes por ainda não existir uma metodologia adequada para ter acesso a essas informações.

Outra limitação está na abrangência do estudo, pois foi analisada apenas a primeira instância da justiça estadual e trabalhista, além da não utilização dos dados por categoria de processo (civil, penal, administrativo) ou de deliberação judicial (liminar, despachos ou sentenças definitivas). Isso impede de obter um olhar mais orgânico, completo, e, consequentemente, uma generalização dos resultados para além da unidade de análise do presente trabalho.

Propõem-se, como agenda para pesquisas futuras, estudos sobre limites e grau de importância que cada variável tem no desempenho dos juízes, para poder identificar os verdadeiros impactos de cada uma. Dessa forma, seria possível possibilitar um direcionamento de propostas e soluções mais adequadas e eficientes para o problema de congestionamento da prestação jurisdicional no Brasil. Outra sugestão é a realização de estudos qualitativos em varas judiciais de maior e menor desempenho, a fim de validar variáveis e evidenciar outras, ausentes nos estudos. Também se propõem estudos para identificar a possibilidade de um modelo funcional de resposta para o desempenho do judiciário que consiga prever os resultados, dados os investimentos e a demanda da sociedade. 


\section{REFERÊNCIAS}

Agresti, A., \& Finlay, B. (2012). Métodos estatísticos para as ciências sociais (4a ed.). Porto Alegre, RS: Penso.

Beenstock, M., \& Haitovsky, Y. (2004). Does the appointment of judges increase the output of the judiciary? International Review of Law and Economics, 24(3), 351-369.

Bielen, S., Peeters, L., Marneffe, W., \& Vereeck, L. (2018). Backlogs and litigation rates: Testing congestion equilibrium across European judiciaries. International Review of Law and Economics, 53, 9-22.

Blank, J. L. T., \& Heezik, A. A. S. (2020). Policy reforms and productivity change in the judiciary system: a cost function approach applied to time series of the Dutch judiciary system between 1980 and 2016. International Transactions in Operational Research, 27(4), 2002-2020.

Christensen, R. K., \& Szmer, J. (2012). Examining the efficiency of the U.S. courts of appeals: Pathologies and prescriptions. International Review of Law and Economics, 32(1), 30-37.

Conselho Nacional de Justiça. (2020). Justiça em números 2020. Brasília, DF: Autor.

Dimitrova-Grajzl, V., Grajzl, P., Slavov, A., \& Zajc, K. (2016). Courts in a transition economy: case disposition and the quantity-quality tradeoff in Bulgaria. Economic Systems, 40(1), 18-38.

Dimitrova-Grajzl, V., Grajzl, P., Sustersic, J., \& Zajc, K. (2012). Court output, judicial staffing, and the demand for court services: evidence from Slovenian courts of first instance. International Review of Law and Economics, 32(1), 19-29.

Falavigna, G., Ippoliti, R., \& Ramello, G. B. (2018). DEA-based Malmquist productivity indexes for understanding courts reform. Socio-Economic Planning Sciences, 62, 31-43.

Fávero, L. P. L., Belfiore, P. P., Silva, F. L., \& Chan, B. L. (2009). Análise de dados: modelagem multivariada de dados para tomada de decisões (6a ed.). Rio de Janeiro, RJ: Elsevier.

Ferro, G., Romero, C. A., \& Romero-Gómez, E. (2018). Efficient courts? A frontier performance assessment. Benchmarking - An International Journal, 25(9), 3443-3458.
Gomes, A. O. (2018). Chegamos ao limite da produtividade no Judiciário brasileiro? In Anais do Encontro da Administração da Justiça, Brasília, DF.

Gomes, A. O., Alves, S. T., \& Silva, J. T. (2018). Effects of investment in information and communication technologies on productivity of courts in Brazil. Government Information Quarterly, 35(3), 480-490.

Gomes, A. O., \& Freitas, M. E. M. (2017). Correlação entre demanda, quantidade de juízes e desempenho judicial em varas da Justiça Federal no Brasil. Revista Direito GV, 13(2), 567-585.

Gomes, A. O., \& Guimarães, T. A. (2013). Desempenho no Judiciário: conceituação, estado da arte e agenda de pesquisa. Revista de Administração Pública, 47(2), 379-401.

Gomes, A. O., Guimaraes, T. A., \& Akutsu, L. (2017). Court caseload management: the role of judges and administrative assistants. Revista de Administração Contemporânea, 21(5), 648-665.

Gomes, A. O., Lopes, L. P. F., Zancan, C., Neto, M. C. L., Costa, A. C. S., Dantas, A. B., \& Ataide, J. A. R. (2017). Variáveis correlacionadas com a produtividade de juízes da primeira instância da Justiça Estadual de Minas Gerais. Sistemas \& Gestão, 12(4), 401-409.

Gujarati, D. N., \& Porter, D. C. (2011). Econometria básica (5a ed.). Porto Alegre, RS: Editora Afiliada.

Hill, R. C., Griffiths, W. E., \& Judge, G. G. (2006). Econometria (2a ed.). São Paulo, SP: Saraiva.

Jonski, K., \& Mankowski, D. (2014). Is sky the limit? Revisiting "exogenous productivity of judges" argument. International Journal for Court Administration, 6(2), 53-72.

Louro, A. C., Santos, W. R., \& Zanquetto, H., Filho. (2018). Productivity antecedents of Brazilian courts of Justice: evidence from Justiça em Números. Brazilian Administration Review, 14(4), 1-18.

Maddala, G. S. (2003). Introdução à econometria (3a ed.). Rio de Janeiro, RJ: LTC.

Peyrache, A., \& Zago, A. (2016). Large courts, small justice! Omega, 64, 42-56.

Procopiuck, M. (2018). Information technology and time of judgment in specialized courts: What is the impact of changing from physical to electronic processing? Government Information Quarterly, 35(3), 491-501. 
Ramseyer, J. M. (2012). Talent matters: judicial productivity and speed in Japan. International Review of Law and Economics, 32(1), 38-48.

Ribeiro, M. C. P., \& Rudiniki, R., Neto. (2016). Uma análise da eficiência do poder judiciário com base no pensamento de Douglas North. Revista Quaestio Iuris, 9(4), 2025-2040.

Rosales-López, V. (2008). Economics of court performance: an empirical analysis. European Journal of Law and Economics, 25(3), 231-251.

Roussey, L., \& Soubeyran, R. (2018). Overburdened judges. International Review of Law and Economics, $55,21-32$.

Santos, S. P., \& Amado, C. A. F. (2014). On the need for reform of the Portuguese judicial system: does data envelopment analysis assessment support it? Omega, 47, 1-16.

Sousa, M. D. M., \& Guimarães, T. A. (2014). Inovação e desempenho na administração judicial: desvendando lacunas conceituais e metodológicas. Review of Administration and Innovation, 11(2), 321.
Sousa, M. D. M., \& Guimaraes, T. A. (2018). Resources, innovation and performance in labor courts in Brazil. Revista de Administração Pública, 52(3), 486-506.

Teixeira, J. C., Bigotte, J. F., Repolho, H. M., \& Antunes, A. P. (2019). Location of courts of justice: the making of the new judiciary map of Portugal. European Journal of Operational Research, 272(2), 608-620.

Voigt, S. (2012). On the optimal number of courts. International Review of Law and Economics, 32(1), 49-62.

Werkema, M. C. C. (1996). Análise de regressão: como entender o relacionamento entre as variaveis de um processo. Belo Horizonte, MG: UFMG.

Wooldridge, J. M. (2010). Introdução a econometria: uma abordagem moderna. São Paulo, SP: Cengage Learning.

Yeung, L. L., \& Azevedo, P. F. (2011). Measuring efficiency of Brazilian courts with data envelopment analysis (DEA). IMA Journal of Management Mathematics, 22(4), 343-356.

\section{Renan Marçal Manzi}

https://orcid.org/0000-0002-4700-8048

Mestre em Administração pela Universidade Federal de Goiás (UFG).E-mail: renanmanzi@outlook.com

\section{Marcos de Moraes Sousa}

https://orcid.org/0000-0002-0901-0550

Doutor em Administração pela Universidade de Brasília (UnB); Professor do Instituto Federal Goiano Campus Ceres (ProfEPT) e do Programa de Pós-Graduação da Universidade Federal de Goiás (PPGADM-UFG).

E-mail: marcos.moraes@ifgoiano.edu.br 